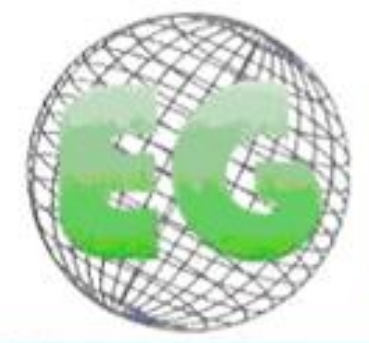

$N^{\circ} 44$

\title{
El método buttonhole como técnica de punción de la fístula arteriovenosa en hemodiálisis: Una revisión de la literatura
}

The buttonhole method like puncture technical of arteriovenous fistula in hemodialysis. A literature review

\author{
*Amador-Marín, Bárbara *Martínez-Montilla, José Manuel

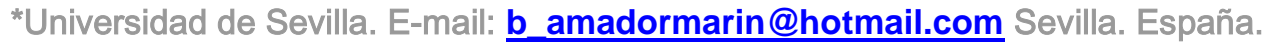

Palabras clave: Diálisis Renal; Fístula Arteriovenosa; Enfermería.

Keywords: Renal Dialysis; Arteriovenous Fistula; Nursing.

\section{RESUMEN}

Introducción: La hemodiálisis es el tratamiento de elección en los pacientes con insuficiencia renal crónica. La clave para un tratamiento adecuado es tener un buen acceso vascular. Por ello, las fístulas arteriovenosas son consideradas la mejor opción. Uno de los principales factores que influyen en la supervivencia de estas es la técnica de punción realizada. El objetivo principal del estudio fue conocer el método buttonhole como técnica de punción de la fístula arteriovenosa en la hemodiálisis.

Metodología: Se realizó una revisión de la literatura científica a través de las siguientes bases de datos: PubMed/Medline, Scopus, Cinahl y Cuiden Plus, desde 2011 hasta 2016. Además, se realizó una búsqueda secundaría manual.

Resultados: Las estrategias iniciales de búsquedas identificaron un total de 84 resultados, siendo finalmente seleccionados 7 estudios. Los resultados encontrados han sido muy diversos, encontrándose resultados para el dolor, tasas de infección, tiempo de hemostasia, dilataciones aneurismáticas, flujo sanguíneo, presión venosa, número de intentos de canulación, seguridad, estética del brazo portador de la fístula y calidad de vida del paciente.

Conclusiones: El método buttonhole parece ser una buena alternativa de punción, ya que está prácticamente exenta de las complicaciones habituales existentes en otras técnicas, como extravasaciones, hematomas, aneurismas, entre otras. Además, facilita la auto-punción en los domicilios. Por otro lado, es necesario un buen aprendizaje previo a la incorporación de esta técnica y un periodo de adaptación por parte de los profesionales, requiriendo implicación por parte de los mismos y del centro.

\section{ABSTRACT}

Introduction: Hemodialysis is the treatment of choice in patients with chronic renal failure. The key to proper treatment is having a good vascular access. Therefore, arteriovenous fistulas are considered the 
best option. One of the main factors that influence the survival of these is the technique of puncture performed. The main objective of the study was to know the method buttonhole like puncture technique arteriovenous fistula in hemodialysis.

Methods: A review of the scientific literature was conducted through the following databases: PubMed/Medline, Scopus, Cinahl and Cuiden Plus, from 2011 to 2016. In addition, a secondary manual search was conducted.

Results: The initial search strategies identified a total of 84 results that finally being selected 7 studies. The results have been mixed, finding results for pain, infection rates, hemostasis time, aneurysmal dilations, blood flow, venous pressure, number of cannulation attempts, security, aesthetics of the carrier arm of the fistula and quality of life of the patient.

Conclusions: The method buttonhole seems to be a good alternative lancing because it is substantially free of existing common complications in other techniques, like extravasations, hematomas and aneurysms, among others. It also facilitates self-lancing at home. Furthermore, a good prior learning incorporating this technique is needed and a period of adaptation by professionals, requiring involvement by them and the center.

\section{INTRODUCCIÓN}

La insuficiencia renal crónica consiste en una destrucción progresiva e irreversible de las nefronas, que afecta a su vez a algunas de las siguientes funciones: filtración, reabsorción o secreción. Esta pérdida progresiva de nefronas causa la hipertrofia de las nefronas sanas, ya que estas últimas tienen que sustituir la función de las nefronas dañadas. Esta situación produce un aumento del flujo, de la presión glomerular y de la filtración en dichas nefronas, provocando su destrucción ${ }^{1}$.

Una vez llegados a este punto de la enfermedad es necesario comenzar con un tratamiento renal sustitutivo (TRS). En España, el $89 \%$ de los pacientes que se inician con el TRS lo hacen a través de la hemodiálisis $(\mathrm{HD})^{2,3}$.

En los últimos años, el envejecimiento de la población hace que los pacientes que se inician en la HD sean personas mayores de 60 años ${ }^{4,5}$. Esta nueva situación trae asociada un mayor número de patologías, entre ellas las vasculares, que contribuyen a un peor estado vascular ${ }^{2,6}$.

La clave para un adecuado tratamiento de HD es tener un buen acceso vascular (AV). Para ello, el AV debe ser canalizado de forma segura y reiterada, debe proporcionar un flujo suficiente y debe tener una baja incidencia de complicaciones ${ }^{7,8}$. Por ello, las fístulas arteriovenosas (FAV) son consideradas la mejor opción ${ }^{3,7,9}$.

Uno de los principales factores que influye en la supervivencia de la FAV es la técnica de punción que se utilice para canalizarla. Ya que un paciente que esté en tratamiento de HD recibe un mínimo de 6 punciones a la semana, es decir, 312 punciones anuales. Esta situación acaba alterando la morfología y las características del vaso sanguíneo $^{10}$. Las diferentes técnicas utilizadas son: "punción en escalera", "área de punción" y "método ojal o buttonhole $(\mathrm{BH})$ ".

La técnica "punción en escalera" distribuye equitativamente las punciones a lo largo de toda la fístula, requiere de un trayecto venoso muy bien desarrollado, produce más dolor y punciones infructuosas. La técnica "área de punción" distribuye las punciones en un área concreta $(2-3 \mathrm{~cm})$, provocando una mayor morbimortalidad del acceso debido a la formación de dilataciones y estenosis. Sin embargo, es la técnica más utilizada, ya que produce menos dolor y punciones infructuosas. Y por último, el 
"método BH" con el cual se realiza la punción en un único punto, con el mismo ángulo y la misma profundidad en cada sesión, creando de esta manera un túnel subcutáneo que facilite la punción y minimice las complicaciones. Sin embargo, en España, la técnica más utilizada es la de "área de punción" y la menos conocida es el método $\mathrm{BH}^{10,11}$.

Por todo ello, nuestro objetivo principal es conocer con mayor profundidad el método $\mathrm{BH}$ como técnica de punción en la HD.

\section{METODOLOGÍA}

Se realizó una revisión de la literatura científica existente, siguiendo las indicaciones del Manual Cochrane ${ }^{12}$ y las recomendaciones del informe PRISMA ${ }^{13}$. A fin de reunir toda la evidencia empírica disponible para responder una pregunta específica de investigación y minimizar sesgos. $Y$ de esta forma, aportar unos resultados más fiables y poder extraer conclusiones.

Las búsquedas de los estudios publicados, se llevaron a cabo desde 2011 hasta 2016, en las siguientes bases de datos electrónicas españolas e internacionales: PubMed/MEDLINE, Scopus, Cinahl, Cuiden Plus. También se realizó una búsqueda secundaría manual en las lista de referencias de los artículos seleccionados. Siendo el tópico de búsqueda, el método BH como técnica de punción en la HD.

Para establecer los descriptores que se emplearon en las búsquedas, se utilizaron los Descriptores de las Ciencias de la Salud (DeCS) y los "Medical Subject Headings" (MESH). Los operadores booleanos utilizados fueron la intersección (AND) y el operador sumatorio (OR), para establecer las operaciones lógicas entre los conceptos.

Los descriptores manejados fueron:

- En Inglés: "Renal Dialysis"; "Kidney Renal”; "Arteriovenous Fistula"; "Nursing".

- En Español: "Diálisis Renal"; "Hemodiálisis"; "Fístula Arteriovenosa"; "Enfermería".

En la tabla 1 se exponen las estrategias de búsqueda utilizadas en cada una de las bases de datos, realizadas en febrero de 2016.

Tabla I. Estrategias de búsqueda realizadas en las bases de datos.

\begin{tabular}{|c|c|}
\hline $\begin{array}{l}\text { BASE DE } \\
\text { DATOS }\end{array}$ & DESCRIPTORES Y LÍMITES \\
\hline PUBMED & $\begin{array}{l}\text { "Renal Dialysis" [All Fields] OR "Kidney Renal" [All Fields]) AND "Arteriovenous } \\
\text { Fistula" [All Fields] AND ("nursing" [Subheading] OR "nursing" [All Fields] OR } \\
\text { "nursing" [MeSH Terms] OR "nursing" [All Fields] OR "breast feeding" [MeSH Terms] } \\
\text { OR ("breast" [All Fields] AND "feeding" [All Fields]) OR "breast feeding" [All Fields]) } \\
\text { AND ("2011/02/23" [PDat]]: "2016/02/21" [PDat] AND (English [lang] OR Portuguese } \\
\text { [lang] OR Spanish [lang])) }\end{array}$ \\
\hline SCOPUS & $\begin{array}{l}\text { TITLE-ASB-KEY (“Renal Dialysis" OR "Kidney Renal") AND TITLE-ABS-KEY } \\
\text { ("Arteriovenous Fistula) AND TITLE-ABS-KEY (“Nursing”) AND PUBYEAR >2010 } \\
\text { AND (LIMIT-TO (LANGUAGE, "English”) OR LIMIT-TO (LANGUAGE, "Portuguese”) } \\
\text { OR LIMIT-TO (LANGUAGE, "Spanish") }\end{array}$ \\
\hline CINAHL & $\begin{array}{l}\text { ("Renal Dialysis OR "Kidney Renal" OR Hemodialysis) AND Arteriovenous Fistula } \\
\text { AND Nursing. Limits: 01/01/2011-31/12/2015 AND English OR Spanish OR Portuguese. }\end{array}$ \\
\hline
\end{tabular}


CUIDEN PLUS (("Hemodiálisis") AND ("Fístula Arteriovenosa") AND ("Enfermería")). Limitado: >

Fuente: Elaboración propia.

Para realizar esta revisión se ha seguido una serie de etapas: 1. Definición del tema. 2. Definición de la pregunta de investigación. 3. Especificación de los criterios de inclusión y exclusión. 4. Elaboración del plan de trabajo para realizar la búsqueda de la literatura por pares. 5. Selección y recuperación de los estudios que cumplen los criterios. 6. Valoración por pares de la calidad metodológica de los estudios. 7. Extracción de los datos. 8. Análisis y síntesis de los resultados. 9. Formulación de las conclusiones $^{14-17}$.

Se seleccionaron los trabajos que cumpliesen con los siguientes criterios de inclusión: 1. Artículos originales y revisiones sistemática relacionados con el tópico de estudio.

2. Idioma: inglés, español y portugués. 3. Fecha de publicación entre 2011-2016. 4. Estudios cualitativos que tengan como afirmativa las dos primeras preguntas de la herramienta de lectura crítica CASPe [Critical Appraisal Skills Programme] ${ }^{18}$. 5. Para los estudios cuantitativos con una calidad de moderada a fuerte en el instrumento EPHPP (Effective Public Health Practice Project) ${ }^{19}$. 6. Para estudios de revisión sistemática se comprobaron los 27 ítems a incluir en una revisión por el informe PRISMA $^{13}$, considerándose apto para la incorporación al estudio aquellos que obtuvieron un $70 \%$ o más de los ítems aportados.

Se excluyeron aquellos artículos con imposibilidad de acceder a texto completo.

Tras las búsquedas, se procedió a la eliminación de artículos que no cumplían con los criterios de inclusión, a través de la lectura del título, resumen y texto completo de los trabajos potencialmente relevantes. A continuación se realizó la calidad metodológica por pares a los artículos incluidos a texto completo, eliminándose aquellos que no cumpliesen con los criterios establecidos por los instrumentos de calidad metodológica.

\section{RESULTADOS}

Las estrategias iniciales de búsquedas identificaron un total de 84 resultados, las cuales fueron objeto de sucesivos cribados, por dos revisores independientes, conforme a los objetivos de la revisión. Finalmente fueron seleccionados 7 estudios (Figura 1).

Figura 1. Diagrama de flujo. Resultados de las búsquedas bibliográficas.

\begin{tabular}{|c|c|}
\hline \multicolumn{2}{|c|}{$\begin{array}{l}\text { Artículos encontrados en Bases de Datos } \\
\text { ( } \mathbf{N} \text { total }=84)\end{array}$} \\
\hline $\begin{array}{l}\text { - Cuiden Plus }(\mathrm{N}=17) \\
\text { - Scopus }(\mathrm{N}=21)\end{array}$ & $\begin{array}{l}\text { - PubMed }(\mathrm{N}=23) \\
\text { - Cinahl }(\mathrm{N}=23)\end{array}$ \\
\hline $\begin{array}{r}\text { Títulos y Resú } \\
\text { (N to }\end{array}$ & $\begin{array}{l}\text { nes revisados } \\
=14)\end{array}$ \\
\hline $\begin{array}{l}\text { - Cuiden Plus }(\mathrm{N}=3) \\
\text { - Scopus }(\mathrm{N}=2)\end{array}$ & $\begin{array}{l}\text { - PubMed }(\mathrm{N}=3) \\
\text { - Cinahl }(\mathrm{N}=6)\end{array}$ \\
\hline
\end{tabular}




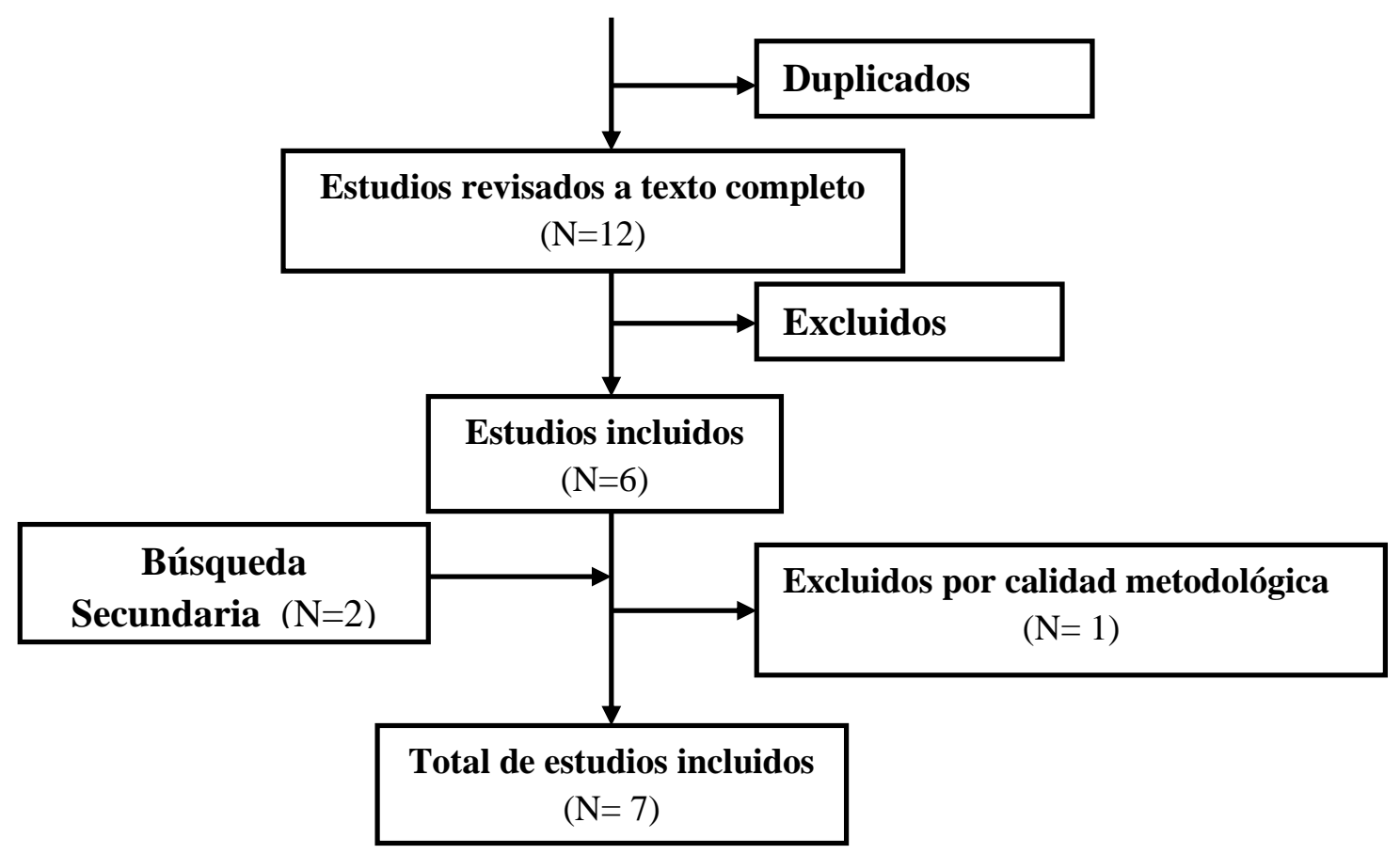

Fuente: Elaboración propia.

De los 7 estudios analizados, 5 utilizaron una metodología cuantitativa, otro una metodología cualitativa, y en otro de los estudios utilizaron una simbiosis de metodología cuantitativa y revisión sistemática. De los estudios cuantitativos, 3 de ellos utilizaron un diseño de cohorte, 1 un diseño descriptivo, 1 es un ensayo clínico y otro un estudio de casos y controles. Por otro lado, el único estudio cualitativo utilizó un diseño descriptivo.

En cuanto al idioma, 4 estudios estaban publicados en inglés, 2 en español y 1 en portugués. Además, 2 de estos estudios fueron desarrollados en Europa, 2 en Oceanía, 2 en América y uno en Asia.

En la tabla II se muestran las características de los estudios incluidos, basadas en el Manual Cochrane ${ }^{12}$. En ella se incluyen los siguientes apartados: autor y año, objetivo del estudio, diseño y duración, muestra, metodología, principales resultados y calidad metodológica de dichos estudios.

Tabla II. Características de los estudios incluidos y valoraciones de los instrumentos de calidad según el tipo de estudio.

\begin{tabular}{|c|c|c|c|}
\hline $\begin{array}{c}\text { Autor y año / } \\
\text { Calidad }\end{array}$ & $\begin{array}{c}\text { Objetivo/ Diseño/ } \\
\text { Muestra }\end{array}$ & Metodología & Resultados \\
\hline $\begin{array}{l}\text { Melo et al., } \\
2015^{20} \text {. } \\
{ }^{*} \text { CASPe: } 8 .\end{array}$ & $\begin{array}{l}\text { - Objetivo: Investigar los } \\
\text { beneficios de la técnica de } \\
\text { BH para canalizar la FAV. } \\
\text { - Diseño: Estudio } \\
\text { cualitativo (descriptivo- } \\
\text { exploratorio). } \\
\text { - Duración: Mayo } 2014 . \\
\text { - Muestra: } 15 \text { pacientes en } \\
\text { HD. } \\
\text { - Edad: } 20-60 \text { años. } \\
\text { - País: Brasil. }\end{array}$ & $\begin{array}{l}\text { Se realizaron entrevistas } \\
\text { siguiendo la estructura de } \\
\text { un cuestionario con } \\
\text { preguntas abiertas. } \\
\text { Se identificaron cuatro } \\
\text { temas para el análisis de } \\
\text { contenido: dolor; } \\
\text { seguridad y calidad; } \\
\text { estética; y bienestar. }\end{array}$ & $\begin{array}{l}\text { - La técnica de } \mathrm{BH} \\
\text { contribuyó de forma } \\
\text { significativa a mejorar la } \\
\text { calidad de vida de los } \\
\text { pacientes. } \\
\text { - Esta técnica se asocio a } \\
\text { un bajo nivel de dolor; a } \\
\text { más seguridad y calidad, a } \\
\text { la preservación de la } \\
\text { estética y al bienestar. }\end{array}$ \\
\hline
\end{tabular}




\begin{tabular}{|c|c|c|c|}
\hline $\begin{array}{l}\text { González et } \\
\text { al., } 2013^{21} \text {. } \\
{ }^{\star *} E P H P P \text { : } \\
\text { Moderada. }\end{array}$ & $\begin{array}{l}\text { - Objetivo: Conocer el } \\
\text { grado de presencia de la } \\
\text { técnica BH para el AV en } \\
\text { las unidades de HD } \\
\text { españolas. } \\
\text { - Diseño: Estudio } \\
\text { cuantitativo (descriptivo- } \\
\text { transversal). } \\
\text { - Duración: no se } \\
\text { especifica. } \\
\text { - Muestra: } 135 \text { unidades de } \\
\text { HD españolas. } \\
\text { - País: España. }\end{array}$ & $\begin{array}{l}\text { Se desarrollo un } \\
\text { cuestionario semi-cerrado, } \\
\text { que fue enviado a } 436 \\
\text { unidades de HD. La tasa } \\
\text { de respuesta fue }>31 \% \\
\text { (131 respuestas). }\end{array}$ & $\begin{array}{l}\text { - El conocimiento del BH es } \\
\text { teórico, pero no lo } \\
\text { suficiente para } \\
\text { implementarlo en las } \\
\text { unidades. } \\
\text { - Su utilización está } \\
\text { parcialmente condicionada } \\
\text { a la opinión y/o } \\
\text { conocimiento de los } \\
\text { nefrólogos. } \\
\text { - Es necesario realizar } \\
\text { talleres prácticos para } \\
\text { aumentar la utilización del } \\
\text { método BH. }\end{array}$ \\
\hline $\begin{array}{l}\text { Kim et al., } \\
2013^{22} \text {. } \\
{ }^{* *} E P H P P \text { : } \\
\text { Moderada. }\end{array}$ & $\begin{array}{l}\text { - Objetivo: Evaluar la } \\
\text { eficacia de la técnica BH } \\
\text { para canalizar la FAV en } \\
\text { pacientes sometidos a HD. } \\
\text { - Diseño: Estudio } \\
\text { cuantitativo (prospectivo de } \\
\text { cohorte). } \\
\text { - Duración: Mayo } 2009 \text { - } \\
\text { Enero } 2010 . \\
\text { - Muestra: } 32 \text { pacientes } \\
\text { con FAV. } \\
\text { - Edad: } 62,4 \text { años. } \\
\text { - País: Corea del Sur. }\end{array}$ & $\begin{array}{l}\text { Fueron analizados el } \\
\text { método BH y el método } \\
\text { "punción en escalera. } \\
\text { Se evaluó la tasa de } \\
\text { acceso vascular (BFR), } \\
\text { presión venosa en la } \\
\text { diálisis (DVP), adecuación } \\
\text { de la diálisis (Kt / V) y } \\
\text { tiempo de coagulación } \\
\text { activado (ACT), el dolor en } \\
\text { la canulación, tiempo de } \\
\text { hemostasias y el estrés de } \\
\text { la enfermera }\end{array}$ & $\begin{array}{l}\text { - No hubo diferencias } \\
\text { estadísticas en ambos } \\
\text { métodos para BFR, DVP, y } \\
\text { la adecuación de la diálisis } \\
\text { entre los dos métodos. } \\
\text { - El tiempo de hemostasia } \\
(P=0,001) \text {, disminución del } \\
\text { dolor durante la canulación } \\
(P=0,001) \text { y el estrés de la } \\
\text { enfermera }(P=0,001) \\
\text { fueron menor en el método } \\
\text { BH. }\end{array}$ \\
\hline $\begin{array}{l}\text { Muir et al., } \\
2^{2013^{23}} \text {. } \\
{ }^{* \star} E P H P P: \\
\text { Moderada. } \\
{ }^{* * *} \text { PRISMA: } \\
19 / 27\end{array}$ & $\begin{array}{l}\text { - Objetivo: Comparar el } \\
\text { método BH y el método } \\
\text { escalera. } \\
\text { - Diseño: Estudio } \\
\text { cuantitativo (retrospectivo) } \\
\text { + revisión sistemática. } \\
\text { - Duración: Enero } 2003 \text { - } \\
\text { Diciembre } 2009 \text {. } \\
\text { - Muestra: } 90 \text { pacientes en } \\
\text { HD. } \\
\text { - Edad: no se especifíca. } \\
\text { - País: Australia. }\end{array}$ & $\begin{array}{l}\text { Estudio cuantitativo: Se } \\
\text { realizó la técnica BH en } 60 \\
\text { pacientes y el método } \\
\text { escalera en } 30 \text {. Se } \\
\text { recogieron datos de todos } \\
\text { los pacientes en HD con } \\
\text { una FAV. } \\
\text { Además se realizó una } \\
\text { revisión sistemática en } \\
\text { diferentes bases de datos } \\
\text { y registros de ensayos } \\
\text { clínicos entre } 1950 \text { y } 2013 \text {. }\end{array}$ & $\begin{array}{l}\text { - Estudio cuantitativo: El } \\
\text { método BH fue asociado a } \\
\text { mayores tasas de } \\
\text { infecciones y necesidades } \\
\text { de apoyo. } \\
\text { - Revisión sistemática: Se } \\
\text { encontró que el método BH } \\
\text { fue asociado a un mayor } \\
\text { riesgo de infecciones } \\
\text { relacionadas con la FAV. }\end{array}$ \\
\hline $\begin{array}{l}\text { Smyth et al., } \\
\mathbf{2 0 1 3}^{24} \text {. } \\
{ }^{\star \star} E P H P P \text { : } \\
\text { Moderada. }\end{array}$ & $\begin{array}{l}\text { - Objetivo: Comparar los } \\
\text { resultados del método BH } \\
\text { y el método escalera. } \\
\text { - Diseño: Estudio } \\
\text { cuantitativo (prospectivo de } \\
\text { cohorte). } \\
\text { - Duración: Octubre - } \\
\text { Diciembre } 2011 . \\
\text { - Muestra: } 104 \text { pacientes } \\
\text { con FAV. } \\
\text { - Edad: > } 18 \text { años. } \\
\text { - País: Australia. }\end{array}$ & $\begin{array}{l}\text { Se evaluó la zona de } \\
\text { punción de las FAV en } \\
\text { todas las sesiones durante } \\
12 \text { semanas. } \\
\text { Se midieron: signos de } \\
\text { infección, formación de } \\
\text { hematoma, formación de } \\
\text { aneurisma e intentos de } \\
\text { canulación. Además se } \\
\text { evaluó el miedo y el dolor. }\end{array}$ & $\begin{array}{l}\text { - No hubo diferencias en la } \\
\text { infección, formación de } \\
\text { hematomas, dolor o miedo } \\
\text { entre ambas técnicas. } \\
\text { - La aparición de aneurisma } \\
\text { fue mayor en el método } \\
\text { escalera. } \\
\text { - El grupo BH requirió más } \\
\text { intentos de canulación. }\end{array}$ \\
\hline $\begin{array}{l}\text { MacRae et } \\
\text { al., 2012 } \\
\\
{ }^{25} \text {. } \\
{ }^{*} E P H P P: \\
\text { Fuerte. }\end{array}$ & $\begin{array}{l}\text { - Objetivo: Comparar el } \\
\text { dolor y las complicaciones } \\
\text { de la FAV con el método } \\
\text { BH y el método escalera. } \\
\text { - Diseño: Estudio } \\
\text { Cuantitativo (ECCA). } \\
\text { - Duración: } 1 \text { año y } 8 \\
\text { meses. } \\
\text { - Muestra: } 140 \text { pacientes } \\
\text { en HD. } \\
\text { - Edad: no se especifica. }\end{array}$ & $\begin{array}{l}\text { - En cada sesión de HD } \\
\text { durante } 8 \text { semanas, se } \\
\text { obtuvieron datos sobre la } \\
\text { presencia y el tamaño de } \\
\text { los hematomas, grado de } \\
\text { dificultad en la punción } \\
\text { percibida por enfermería, } \\
\text { signos de infección y } \\
\text { tiempo de hemostasia. } \\
\text { - Seguimiento de } 1 \text { años } \\
\text { para evaluar la }\end{array}$ & $\begin{array}{l}\text { - A las } 8 \text { semanas el dolor } \\
\text { fue similar en ambos } \\
\text { grupos. } \\
\text { - Mayor formación de } \\
\text { hematomas en método } \\
\text { escalera. } \\
\text { - Menor signo de infección } \\
\text { localizada en el método BH. } \\
\text { Pero hubo varios casos de } \\
\text { bacteriemia en método BH. } \\
\text { - Mayor riesgo de infección }\end{array}$ \\
\hline
\end{tabular}




\begin{tabular}{|c|c|c|c|}
\hline & - País: Canadá. & $\begin{array}{l}\text { permeabilidad de la FAV y } \\
\text { complicaciones } \\
\text { infecciosas. }\end{array}$ & $\begin{array}{l}\text { en uso rutinario del método } \\
\mathrm{BH} \text {. }\end{array}$ \\
\hline $\begin{array}{l}\text { Grau et al., } \\
2011^{10} \text {. } \\
{ }^{* *} E P H P P: \\
\text { Moderada. }\end{array}$ & $\begin{array}{l}\text { - Objetivo: Introducción del } \\
\text { método BH en una Unidad } \\
\text { de HD. } \\
\text { - Diseño: Estudio } \\
\text { cuantitativo (descriptivo } \\
\text { observacional). } \\
\text { - Duración: } 6 \text { meses. } \\
\text { - Muestra: } 35 \text { pacientes en } \\
\text { HD. } \\
\text { - Edad: no se especifica. } \\
\text { - País: España. }\end{array}$ & $\begin{array}{l}\text { - El personal de } \\
\text { enfermería se formó en la } \\
\text { técnica y se creó un } \\
\text { protocolo de punción. } \\
\text { - Seguimiento de la } \\
\text { técnica a través de: } \\
\text { examen físico de la FAV, } \\
\text { estado del túnel, } \\
\text { características de la } \\
\text { punción, dolor percibido } \\
\text { (EVA), parámetros } \\
\text { habituales durante la } \\
\text { sesión, complicaciones, } \\
\text { tiempo de hemostasia y } \\
\text { mapa del AV. }\end{array}$ & $\begin{array}{l}\text { - Disminución del dolor, } \\
\text { tiempo de hemostasia y } \\
\text { tamaño de aneurismas } \\
\text { producidas por la técnica. } \\
\text { - Muchos pacientes optan } \\
\text { por la auto-punción. } \\
\text { - Los resultados de la } \\
\text { encuesta de satisfacción } \\
\text { fueron positivos, dando } \\
\text { como motivos: reducción } \\
\text { del dolor, ansiedad ante la } \\
\text { punción, complicaciones y } \\
\text { del tiempo de hemostasia. }\end{array}$ \\
\hline \multicolumn{4}{|c|}{$\begin{array}{l}\text { BH = Buttonhole; FAV = Fístula Arteriovenosa; AV = Acceso Vascular; HD = Hemodiálisis; ECCA = Ensayo Clínico } \\
\text { Controlado y Aleatorizado; EVA = Escala Visual Analógica. } \\
{ }^{*} \text { CASPe (Critical Appraisal Skills Programme): Programa de lectura crítica para la valoración de calidad } \\
\text { metodológica de estudios cualitativos. }{ }^{* \star} \text { EPHPPP (Effective Public Health Practice Project): Instrumento de medición } \\
\text { de calidad de los estudios cuantitativos. }{ }^{\star \star \star} \text { PRISMA. }\end{array}$} \\
\hline
\end{tabular}

Fuente: Elaboración propia, basada en el Manual Cochrane.

En cuanto a la calidad metodológica de los estudios incluidos podemos decir que 5 de los estudios cuantitativos tienen una calidad metodológica moderada ${ }^{10,21-24}$ y uno de ellos una calidad fuerte ${ }^{25}$ a través del instrumento EPHPP ${ }^{19}$. El único estudio cualitativo obtuvo una puntuación de 8 sobre $10^{20}$ en la herramienta CASPe ${ }^{18}$ y el estudio de revisión obtuvo 19 ítems de $27^{23}$ según el informe PRISMA ${ }^{13}$.

En nuestros resultados hemos encontramos diversidad en cuanto al objetivo de estudio relacionado con el método $\mathrm{BH}, 3$ estudios han comparado este método de punción con el método en escalera ${ }^{23-25}, 2$ investigan los beneficios y la eficacia de utilizar el método $\mathrm{BH}$ para canalizar las $\mathrm{FAV}^{20,22}$, 1 describe la experiencia de introducir este método en una unidad de $\mathrm{HD}^{10}$ y otro analiza la presencia de esta técnicas en las unidades de $\mathrm{HD}^{21}$.

Los resultados encontrados en nuestra revisión han sido muy diversos, ya que se han encontrados resultados para el dolor ${ }^{10,20,22,24,25}$, infección ${ }^{10,23-25}$, tiempo de hemostasia $^{10,22}$, dilataciones aneurismáticas ${ }^{10,24}$, flujo sanguíneo del $\mathrm{AV}^{22}$, presión venosa de diálisis ${ }^{22}$, $\mathrm{n}^{\circ}$ de intentos de canulación ${ }^{10,24}$, seguridad ${ }^{10,20,24}$, estética del brazo portador de la $\mathrm{FAV}^{20,24,25}$ y calidad de vida en el paciente ${ }^{20}$.

Referente al dolor experimentado por los pacientes durante la canalización de la FAV a través del método $\mathrm{BH}$, podemos decir que algunos estudios afirman que esta técnica se asocia a una reducción del dolor ${ }^{10,20,22}$, mientras que otros estudios afirman no encontrar diferencias significativas para el dolor entre el método $\mathrm{BH}$ u otro método de punción ${ }^{24,25}$.

En cuanto a las infecciones, también encontramos resultados dispares, ya que en algunos estudios afirman que el método $\mathrm{BH}$ como técnica de punción es asociado a mayores tasas de infección en comparación con el método escalera ${ }^{23,25}$. Sin embargo, en otro estudio no se encontró diferencias estadísticamente significativas para la tasa de infección entre estos dos métodos ${ }^{24}$. 
En nuestros resultados sobre el tiempo de hemostasia y las dilataciones aneurismaticas han sido favorables, ya que los estudios encontrados afirman que el tiempo de hemostasia bajó significativamente gracias a la utilización del método $\mathrm{BH}$ y las dilataciones aneurismáticas fueron menores con este método en comparación con el método escalera ${ }^{10,22}$.

En cuanto al flujo sanguíneo del acceso vascular y a la presión venosa de diálisis, en nuestros resultados no se han encontrado diferencias estadísticamente significativas comparando el método $\mathrm{BH}$ con el método escalera ${ }^{22}$.

Otro resultado encontrado ha sido el referente al no de intentos de canulación a través del método $\mathrm{BH}$. Algunos autores afirman que este método requiere de un mayor número de punciones mientras se está creando el túnel ${ }^{24}$. $Y$ otros autores, afirman que una vez que se crea el túnel la técnica de punción es muy sencilla, por lo que muchos pacientes optan por la auto-punción ${ }^{10}$.

En cuanto a la seguridad que proporciona este método, algunos estudios sugieren que el método $\mathrm{BH}$ como técnica de punción proporciona mayor seguridad y tranquilidad a los pacientes durante la canulación ${ }^{10,22}$. Mientras que otros estudios no han encontrado diferencias estadísticamente significativas para la seguridad entre este método y el método escalera ${ }^{24}$. Por otro lado, algunos autores afirman que el estrés en el personal de enfermería durante la canalización es menor gracias a este método ${ }^{22}$.

En cuanto a la estética del brazo portador de la FAV, algunos estudios afirman que gracias al método $\mathrm{BH}$ la estética del brazo mejora, ya que se forman menos hematomas ${ }^{20,25}$. Sin embargo, en otro estudio encontrado afirman que no existen diferencias estadísticamente significativas en cuanto a la formación de hematomas utilizando el método $\mathrm{BH}$ o el método escalera ${ }^{24}$.

Por último, sólo se ha encontrado un estudio que afirme que la calidad de vida de los pacientes mejora gracias a la utilización del método $\mathrm{BH}$ como método de punción de la $\mathrm{FAV}^{20}$.

Y no se han encontrado estudios que comparen el método BH con el método "área de punción".

\section{DISCUSIÓN}

La evidencia científica coincide en la importancia del cuidado de la FAV y de una adecuada técnica de punción de la misma para conseguir su correcto desarrollo, una adecuada permeabilidad, prolongar su supervivencia y minimizar el riesgo de complicaciones en esta. Ya que las complicaciones derivadas del AV en la HD suponen hasta un $25 \%$ de las hospitalizaciones en los pacientes que se encuentran en TRS. Este hecho supone un elevado índice de morbilidad y un aumento del costo sanitario, además de generar ansiedad y disconfort para el paciente ${ }^{26,27}$. Así mismo, la técnica de punción utilizada para canalizar la FAV es uno de los factores que más influye en la supervivencia de esta. De ahí la importancia de conocer con mayor profundidad el método BH como técnica de punción en la HD.

Con respecto a los hallazgos encontrados en nuestra revisión, estos parecen indicar que la mayoría de los estudios que se están realizando sobre el método $\mathrm{BH}$ se 
centran en los beneficios o en las complicaciones derivadas de la utilización de este método como técnica de punción. Centrándose principalmente en ciertos aspectos, tales como el dolor experimentado por el paciente, tasas de infección, tiempo de hemostasia, formación de aneurismas, no de intentos a la hora de canalizar el acceso, seguridad, estética del brazo portador de la FAV y calidad de vida del paciente.

En cuanto al dolor, nuestros resultados indican la existencia de discrepancias entre los diferentes estudios, ya que algunos autores asocian la utilización de esta técnica con la reducción del dolor experimentado por el paciente ${ }^{10,20,22}$, coincidiendo esta afirmación con la de otros autores ${ }^{26,28,29}$. Mientras que en otros estudios se afirma que el dolor experimentado por los pacientes durante la punción de la FAV a través del método $\mathrm{BH}$ es el mismo que el experimentado con el método escalera ${ }^{24,25}$. Estas discrepancias entre los resultados encontrados pueden ser debidas a la propia naturaleza subjetiva del dolor y a su variabilidad individual ${ }^{30}$.

Algo semejante sucede con los resultados encontrados para las infecciones, ya que algunos autores asocian el método $\mathrm{BH}$ con mayores tasas de infección en comparación con el método escalera ${ }^{10,23,25}$, coincidiendo con los resultados encontrados por otros autores ${ }^{27,31,32}$. Sin embargo, Smith et al. ${ }^{24}$ en su estudio afirmó no encontrar ninguna relación en cuanto a las tasas de infección y la utilización de dicho método.

Además, sería conveniente recalcar que las infecciones, comúnmente, son causadas por falta de atención en la técnica aséptica durante la punción del AV. De ahí, la importancia de reiterar en la existencia de un protocolo de asepsia adecuado en las unidades de HD y su correcto cumplimiento, con el fin de prevenir futuras complicaciones y reducir el coste sanitario derivado de la aparición de dichas complicaciones ${ }^{2,26}$. Además, coincidimos con la afirmación realizada por otros autores, en cuanto a que la mayoría de los estudios publicados no hacen referencia a la técnica de asepsia utilizada durante dicho procedimiento ${ }^{11}$.

Por otro lado, los hallazgos encontrados para la hemostasia y las dilataciones aneurismáticas son favorables, ya que se relaciona el método $\mathrm{BH}$ con una disminución del tiempo de hemostasia y con menos formación de aneurismas ${ }^{10,22}$, coincidiendo estos con los encontrados por otros autores ${ }^{29}$. Probablemente, estos resultados positivos sean debido a que una vez que se crea el túnel con el método $\mathrm{BH}$, la punción de la FAV se realiza con una aguja de punta roma, la cual es menos traumática para el AV y produce dilataciones de menor tamaño.

En cuanto al $\mathrm{n}^{\circ}$ de intentos requeridos para canalizar el $\mathrm{AV}$ con el método $\mathrm{BH}$, nuestros resultados concuerdan con los encontrados por otros autores ${ }^{10,11,24,25,28-30}$. Y es que el $n^{\circ}$ de punciones se ve incrementado cuando se está creando el túnel, pero una vez que el túnel está creado las punciones son fáciles y rápidas, favoreciendo incluso la auto-punción Por tanto, la utilización del método BH como técnica de punción, al inicio, supone cierta dificultad para los profesionales sanitarios y para el paciente, ya que requiere de un periodo previo de adaptación, en el cual se debe lograr la experiencia y habilidad necesaria para que la técnica resulte fácil.

Acerca de la formación de hematomas, nuestros resultados son dispares, ya que algunos estudios han relacionado el método $\mathrm{BH}$ con la formación de menos hematomas y por tanto mejora la estética del brazo portador de la $F A V^{20,25}$. Este hecho, coincide con la afirmación realizada por otros autores ${ }^{31}$. Por el contrario, otros 
autores afirman que la formación de hematomas en el AV es similar utilizando el método $\mathrm{BH}$ o el método escalera ${ }^{24}$.

El siguiente aspecto a tratar hace referencia a que el método $\mathrm{BH}$ como técnica de punción del AV en las Unidades de HD españolas está siendo lento e inferior en comparación con su implantación en otros países europeos y asiáticos ${ }^{11,21}$. Actualmente, en España, la mayoría de centros de HD utilizan la técnica "área de punción", a pesar de la morbilidad que causa en el AV. Sin embargo, no se han encontrado estudios que comparen los resultados del método $\mathrm{BH}$ con los de la técnica "área de punción".

En este sentido, algunos autores afirman que el método $\mathrm{BH}$ es ampliamente conocido en las diferentes unidades de diálisis. Sin embargo, este conocimiento es principalmente teórico y poco profundo. Además, a pesar de ser una técnica enfermera, su utilización está parcialmente condicionada a la opinión y/o conocimiento que los nefrólogos tengan sobre ella ${ }^{21}$.

Hay que mencionar, además, la carga de trabajo y la dificultad que existe para aplicar este método de punción en las unidades de HD españolas. Puesto que debe existir una implicación importante del personal de enfermería, requiriendo un período previo de aprendizaje y cierto grado de disponibilidad y coordinación de turnos, ya que debe ser la misma persona quién realice la punción mientras se crea el túnel ${ }^{11,29}$. Por ello, consideramos imprescindible la colaboración de los supervisores de las unidades de HD para facilitar al personal de enfermería los cambios de turno y rotaciones de días libres para poder llevar a cabo está técnica.

Dicho todo esto, la técnica $\mathrm{BH}$ parece ser una buena opción tanto para el paciente como para los profesionales ${ }^{10,28,33}$, ya que el $n^{\circ}$ de complicaciones en el $\mathrm{AV}$ relacionadas con la utilización de este método son similares a los que aparecen con la utilización del método escalera o inclusive estas se ven disminuidas. Por tanto, la calidad de vida de los pacientes puede mejora gracias a la utilización de este método ${ }^{20}$.

En relación con futuras líneas de investigación, creemos que sería conveniente realizar estudios experimentales donde se compare el método $\mathrm{BH}$ con el método "área de punción", ya que es el método más utilizado a nivel nacional. Además, los estudios que se realicen en un futuro deberían tener un periodo de seguimiento más largo y contar con una muestra mayor. Así como se debería mejorar los protocolos de asepsia en las unidades de HD para poder reducir al mínimo las posibles casos de infección relacionados con esta técnica.

Referente a las limitaciones de esta revisión, estas podrían estar relacionadas con el sesgo de selección y publicación inherente en toda revisión. Este hecho ha intentado solventarse mediante la utilización de los diferentes instrumentos de calidad. Además, habría que ser prudentes a la hora de interpretar los resultados, debido a su propia heterogeneidad.

\section{CONCLUSIONES}

Por último, podemos concluir que el método $\mathrm{BH}$ es una buena alternativa de punción al estar prácticamente exenta de las complicaciones habituales existentes en otras técnicas, como extravasaciones, hematomas, aneurismas, entre otras. Asimismo, 
facilita la auto-punción en domicilio por los pacientes, gracias a la sencillez de la técnica una vez tunelizado la fistula.

Por otro lado, se requiere practica y experiencia hasta conseguir una buena tunelización de la fistula. Por ello, se cree necesario un buen aprendizaje previo por parte del personal de enfermería para la incorporación de esta técnica de punción, así como un periodo de adaptación por parte de los profesionales, requiriendo implicación por parte de los mismos y del centro. Además, creemos necesario el empoderamiento de las enfermeras a la hora de elegir una técnica de punción u otra, siendo siempre consensuada con el paciente.

\section{REFERENCIAS}

1. Santiago MA, Reina $M$, Fernández $E$, Palazuelos $M$, Roquet $T$, López $A$. Análisis cualitativo de las diferencias básicas en la hemodiálisis pediátrica y adulta. Rev Paraninfo Digital. 2011;V(14). Disponible en: http://www.indexf.com/para/n14/pdf/159d.pdf

2. Ramírez EB, Amador B. Las complicaciones de la hemodiálisis y el papel de enfermería: Revisión bibliográfica. Rev Paraninfo Digital. 2014;VIII(20). Disponible en: http://www.index-f.com/para/n20/255.php

3. Fariñas M, García J, Gutiérrez M. Infecciones asociadas a los catéteres utilizados para la hemodiálisis y la diálisis peritoneal. Enferm Infec Micr Cl. 2008;26(8):51826.

4. Martínez A, Moñux G, Martínez I, Sánchez L, Serrano F. Valor pronóstico de la flujometría intraoperatoria en las fístulas nativas para hemodiálisis. Angiología. 2010;62(3):97-102.

5. Guerra-Martín MD, Amador-Marín B, Martínez-Montilla JM. Problemas de salud de los cuidadores familiares de personas mayores de 65 años afectadas de insuficiencia renal crónica: una revisión sistemática. An Sist Sanit Navar. 2015;38(3):425-38.

6. Pelayo R, Cobo JL, Reyero M, Sáenz de Buruaga A, Tovar A, Alonso R, et al. Repercusión del acceso vascular sobre la calidad de vida de los pacientes en tratamiento con hemodiálisis. Rev Soc Esp Enferm Nefrol. 2011;14(4):242-9.

7. Moyano MJ, Salgueira M, Aresté N, Escalera B, del Toro N, Jiménez-Víbora E, et al. Estudio comparativo del uso de prótesis vasculares de PTFE a nivel de antebrazo vs catéter permanente. Nefrología. 2006;26(5):594-9.

8. Santesteban A, González S, Ochoa B. Manejo y cuidados de enfermería de los accesos vasculares para hemodiálisis. Pulso. 2008;oct(55):33-5.

9. Murphy $F$. The ongoing challenges with renal vascular access. British Journal of Nursing. 2011;20(4 suppl):6-14.

10. Grau C, et al. La punción del acceso vascular en hemodiálisis es una necesidad, el método Buttonhole una opción. Revista Sociedad Española de Enfermería Nefrológica. 2011;14(1):30-6.

11. Baena L, Martín B, Marcos A. Implantación de la técnica del "ojal" o "Buttonhole" en una unidad de hemodiálisis hospitalaria: satisfacción del paciente. Enfermería Nefrológica. 2015;18(1):61-4.

12. Higgins JPT, Green S (Edit.). Cochrane Handbook for Systematic Reviews of Interventions. Version 5.1.0 [Consultado 5 abril 2016]. The Cochrane Collaboration, 2011. Disponible en: www.cochrane-handbook.org.

13. Urrutia G, Bonfill X. Declaración PRISMA: una propuesta para mejorar la publicación de revisiones sistemáticas y metaanálisis. Med Clin (Barc) 2010;135:507-11. 
14. Cué $M$, Díaz G, Díaz AG, Valdés MC. El artículo de revisión. RESUMED. 1996;9(2):86-96.

15. Guerra JA, Martín P, Santos JM. Las revisiones sistemáticas, niveles de evidencia y grados de recomendación. Atención Primaria en la Red [internet]. 2003 [Consultado 5 abril 2016]. Disponible http://www.guiamark.com/images/stories/mbe_rev_sistematicas2.pdf

16. Beltrán OA. Revisiones sistemáticas de la literatura. Asociaciones Colombianas de Gastroenterología, Endoscopia digestiva, Coloproctología y Hepatología. 2005;20(1):60-9.

17. Ferreira I, Urrútia G, Alonso-Coello P. Revisiones sistemáticas y metaanálisis: bases conceptuales e interpretación. Rev Esp Cardiol. 2011;64(8):688-96.

18. Cano A, González T, Cabello JB. Plantilla para ayudarte a entender un estudio cualitativo. en: CASPE. Guías caspe de Lectura Crítica de la Literatura Médica. Alicante: CASPE; 2010. Cuaderno III. p.3-8.

19. Effective Public Health Practice Project. Quality assessment tool for quantitative studies [internet]. [Consultado 5 abril 2016]. Disponible en: http://www.ephpp.ca/pdf/quality\%20assessment\%20tool_2010_2.pdf

20. Melo D, Lírio J, Lavoyer C, Campos H. Satisfação dos pacientes com a técnica de buttonhole. Cogitare Enfermagem. 2015;20(3):483-8.

21. González J, Momblanch T, Moreno C, Sanz A, Navarro L, Martí A, et al. Análisis enfermero de la presencia de la técnica del Ojal en las unidades de hemodiálisis Españolas. Enferm Nefrol. 2013;16(2):93-8.

22. Kim MK, Kim HS. Clinical effects of buttonhole cannulation method on hemodialysis patients. Hemodial Int. 2013;17:294-9.

23. Muir CA, Kotwal SS, Hawley CM, Polkinghorne K, Gallagher MP, Snelling P, et al. Buttonhole Cannulation and Clinical Outcomes in a Home Hemodialysis Cohort and Systematic Review. Clin J Am Soc Nephro. 2014;9(1):110-9.

24. Smyth W, Hartig V, Manickam V. Outcomes of buttonhole and rope-ladder cannulation techniques in a tropical renal service. J Renal Care. 2013;39(3):157-65.

25. MacRae JM, Ahmed SB, Atkar R, Hemmelgarn BR. A Randomized Trial Comparing Buttonhole with Rope Ladder Needling in Conventional Hemodialysis Patients. Clin J Am Soc Nephro. 2012;7:1632-8.

26. Baena L. Button-hole: técnica de punción de elección en hemodiálisis domiciliaria. Enferm Nefrol. 2014;17(2):147-9.

27. Grau C, Vinuesa X, Moya C, García R, Viudes E, Ibeas JA. Buttonhole como técnica de elección para evitar la pérdida del acceso vascular complejo. Enferm Nefrol. 2013;16 Suppl(1):47-9.

28. Fernández IM. Cuidados de la Fístula Arteriovenosa durante la sesión de Hemodiálisis. Rev ENE Enferm. 2013;7(1):1-6.

29. Ludlow V. Buttonhole cannulation in hemodialysis: Improved outcomes and increased expense-Is it worth it?. CANNT Journal. 2010;20(1);29-37.

30. Evans LM. Buttonhole cannulation for haemodialysis: a nursing review. RSA Journal. 2012;8(3):146-51.

31. Atkara RK, MacRae JM. The buttonhole technique for fistula cannulation: pros and cons. Curr Opin Nephrol Hy. 2013;22(6):629-36.

32. Ball L. Fatal Vascular Access Hemorrhage: Reducing the Odds. Nephrol Nurs J. 2013;40(4):297-303.

33. Flynn A, Linton A. Buttonhole cannulation principles. RSA Journal. 2011;7(2):90-3. 
Recibido: 24 de mayo 2016; Aceptado: 23 de agosto 2016 